\title{
Effect of Trait Anxiety on State Anxiety Through Intolerance of Uncertainty and Mastery
}

\author{
Liu Jingyuan ${ }^{1}$, Li Hong, ${ }^{2}$ * \\ ${ }^{1}$ Center for Psychological Development, Tsinghua University, Beijing, China \\ ${ }^{2}$ Department of Psychology, Tsinghua University, Beijing, China
}

\section{Email address:}

liujy2020@mail.tsinghua.edu.cn (Liu Jingyuan), lhong@mail.tsinghua.edu.cn (Li Hong)

${ }^{*}$ Corresponding author

\section{To cite this article:}

Liu Jingyuan, Li Hong. Effect of Trait Anxiety on State Anxiety Through Intolerance of Uncertainty and Mastery. Science Innovation. Vol. 9, No. 4, 2021, pp. 134-138. doi: 10.11648/j.si.20210904.13

Received: April 7, 2021; Accepted: May 10, 2021; Published: May 24, 2021

\begin{abstract}
Background: Few studies have explored the relationship between trait anxiety, intolerable of uncertainty, mastery and state anxiety, which is of great significance to understand the causes and relief of anxiety. Objective: To explore the relationship among trait anxiety, intolerance of uncertainty, mastery and state anxiety. Methods: Ninety-four college students were conveniently chosen to engage in a survey research including Trait Anxiety Inventory, Mastery Scale, the self-evaluation of intolerance of uncertainty and state anxiety. Results: (1) Correlation analysis showed that, the relationships between each pair of trait anxiety, intolerance of uncertainty and state anxiety were significantly positive. Trait anxiety, intolerance of uncertainty and state anxiety were all negatively related to mastery. (2) Mediating effect analysis showed that, intolerance of uncertainty and mastery played a partial mediating role between trait anxiety and state anxiety respectively (the ratio of the mediating effect to total effects was $9.10 \%$ and $10.53 \%$ respectively). (3) Chain mediating effect analysis showed that, the chain mediating effect of intolerance of uncertainty and mastery between trait anxiety and state anxiety was significant (3.89\%). Conclusion: Trait anxiety affected state anxiety not only directly but through the chain mediating roles of intolerance of uncertainty and mastery.
\end{abstract}

Keywords: Trait Anxiety, Intolerance of Uncertainty, Mastery, State Anxiety

\section{特质焦虑通过无法忍受不确定性及掌控感对状态焦虑的作用}

\author{
刘静远 ${ }^{1}$, 李虹 ${ }^{2 *}$ \\ 1清华大学学生心理发展指导中心, 北京, 中国 \\ 2清华大学心理学系, 北京, 中国
}

邮箱

liujy2020@mail.tsinghua.edu.cn（刘静远）, lhong@mail.tsinghua.edu.cn（李虹）

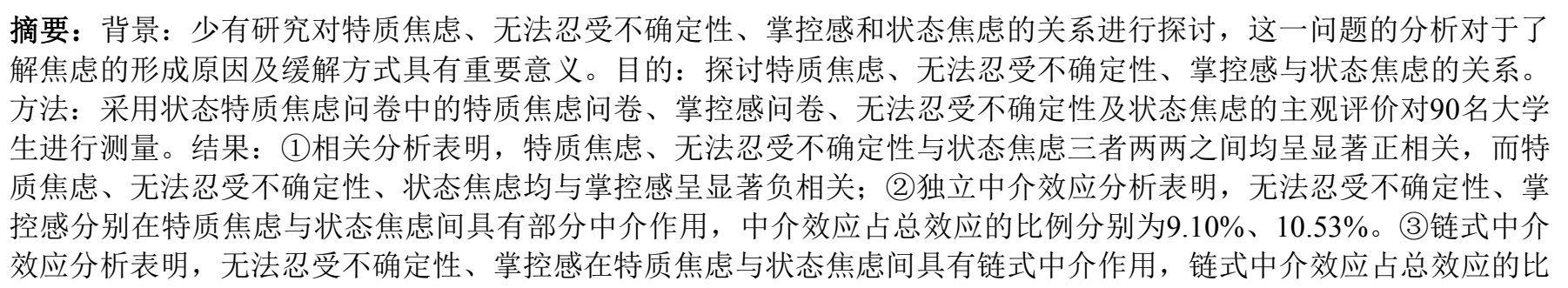


例为 $3.89 \%$ 。结论: 特质焦虑既通过直接路径, 也通过无法忍受不确定性 $\rightarrow$ 掌控感的链式中介作用这一间接路径影响 个体的状态焦虑。

关键词：特质焦虑，无法忍受不确定性，掌控感，状态焦虑

\section{1. 引言}

焦虑 (anxiety) 是个体对即将来临的可能会造成危险 或威胁的情境产生的紧张、不安、忧虑、烦恼等不愉快的 复杂情绪反应[1-3]。焦虑涉及到对无望感及潜在威胁的不 可控制感的认知, 包括对未来忧虑的感知, 对潜在威胁的 警觉和应对潜在威胁的长期准备状态 [4, 5]。Spielberger将 焦虑分为状态焦虑与特质焦虑。其中, 状态焦虑 (state anxiety) 是人们在特定情境中所产生的特定反应状态 (如 紧张、恐惧、忧虑), 常常伴有植物神经系统功能的改变 (如心跳加快、血压升高、呼吸急促) ; 而特质焦虑 (trait anxiety) 则是一种稳定的焦虑倾向, 持续表现出状态焦虑 的特征。具体而言, 状态焦虑是一种较为短暂的情绪状态, 可随着情境的转移和时间的推移而逐步减缓直至消失。状 态焦虑的紧张度和起伏性会自然地发生变化, 也可以通过 情境刺激的方式加以启动; 特质焦虑则是一种相对稳定的 人格特质, 与先天生物学基础以及习得有关的焦虑倾向。 特质焦虑的个体倾向于把外界广泛的刺激环境知觉为危 险的或威胁性的, 并因此对这种危险或威胁产生上述状态 焦虑反应[6]。除特质焦虑外, 无法忍受不确定性及掌控感 也被认为是影响状态焦虑的重要因素。

无法忍受不确定性 (intolerance of uncertainty) 是指 个体面对不确定的情境或事件时产生的一系列认知偏差, 这一认知偏差使个体倾向于认为不确定的情境或事件是 无法忍受且应该回避的, 而不管该情境或事件发生的可能 性有多大和导致的结果如何 $[7,8]$ 。研究表明, 无法忍受不 确定性对焦虑具有直接影响 [9-12], 而无法忍受不确定性 的降低可以有效缓解广泛性焦虑障碍患者的症状 $[13,14]$ 。

掌控感 (mastery) 又称自我控制感 (perceived control), 是个体认为自我能够对生活和周围环境施加影 响和控制的程度 $[15,16]$ 。许多研究发现失控感会伴随焦虑 感 $[5,17,18]$ 。个体所知觉到的情绪控制的缺失, 是导致和 加重个体焦虑障碍的重要因素 $[19,20]$ 。也有研究发现个体 的掌控感感水平越高, 焦虑水平就越低[21]。

综上, 特质焦虑、无法忍受不确定性以及掌控感分别 从不同方面影响状态焦虑。然而, 它们之间的关系是怎样 的? 特质焦虑作为一种相对稳定的人格特质是否会通过 无法忍受不确定性和掌控感影响状态焦虑? 迄今为止的 文献中很少有研究对这一问题进行探讨。根据上述已有研 究发现[9-12, 19-21], 我们推测: 无法忍受不确定性、掌 控感在特质焦虑与状态焦虑之间分别具有中介作用; 同时 存在两种可能成立的链式中介路径: (1) 特质焦虑 $\rightarrow$ 无法 忍受不确定性 $\rightarrow$ 掌控感 $\rightarrow$ 状态焦虑; (2) 特质焦虑 $\rightarrow$ 掌控 感 $\rightarrow$ 无法忍受不确定性 $\rightarrow$ 状态焦虑。本研究试图采用中介 效应分析策略对其加以分析验证, 以确认特质焦虑如何通 过无法忍受不确定性和掌控感影响状态焦虑, 从而揭示特
质焦虑影响状态焦虑的内在方式, 并且为焦虑的预防和干 预实践提供策略性参考。

\section{2. 对象与方法}

\section{1. 对象}

采用方便取样, 三天实验时间内招募北京市某高校 90 名大学生为研究对象。其中男 39 名, 女51名, 所有被试平 均年龄21.92 岁 $(s=3.10)$ 。

\section{2. 工具}

\subsection{1. 特质焦虑的测量}

采用Spielberger编制的状态-特质焦虑问卷中的特质 焦虑问卷 (Trait Anxiety Inventory, TAI) 对被试的特质焦 虑进行测量[22]。TAI包括共 20 个条目, 其中 10 个反向条 目, 原始问卷为 4 点计分, 为提高区分度和信效度[23-25], 本研究中采用 1 (几乎没有) 7 (几乎总是如此) 7 点计分, 分数越高表示特质焦虑水平越高。本研究中该问卷的 Cronbach $\alpha$ 系数为 0.912 。

\subsection{2. 无法忍受不确定性的测量}

让被试主观自评此时此刻的无法忍受不确定性 “整个实验过程中充满了不确定性, 我对不确定性的 忍受程度”。本研究中采用 0 (完全可以忍受) 100 (完全无 法忍受) 计分, 分数越高表示无法忍受不确定性的程度越 强。

\subsection{3. 掌控感的测量}

参考Pearlin等人的方式对掌控感进行测量[16]。包括5 个条目: (1) 我对整个实验无法控制; (2) 我真的无法解 决实验过程中提到的问题。(3) 在实验进行过程中我感到 无助。(4) 我觉得自己被刚刚的实验所左右。(5) 刚刚 的实验结果的对错好坏主要取决于我自己。其中前 4 条反 向计分, 本研究中采用 0 (完全不赞同) 100 (完全赞同) 计 分, 取均值后分数越高表示掌控感越强。

\subsection{4. 状态焦虑的测量}

采用视觉模拟心境量表 (Visual Analogue Mood Scales, VAMS) 测量状态焦虑水平[26], 让被试从 0 到 100 之间选择一个数字主观评价“此时此刻的焦虑程度”。

\section{3. 实验程序}

被试通过校内张贴的海报上的问卷星链接进行线上 报名, 进入实验室后, 依次进行特质焦虑的测量、无法 
忍受不确定性的测量、掌控感的测量以及状态焦虑的测 量。

\section{4. 数据处理}

对数据采用多重共线性分析后发现, 方差膨胀因子 (Variance Inflation Factor, VIF) 均小于 10 , 条件指数
(Condition Index, CI) 均小于30, 特征根 (eigenvalue) 均大 于 0 。因此, 本研究数据不存在严重多重共线性, 可以进 行进一步的数据分析。采用SPSS20.0对数据进行描述性统 计和相关性分析, 使用Hayes开发的PROCESS程序进行链 式中介效应分析[27]。

\section{3. 结果}

\section{1. 描述性统计和各变量间相关分析}

表1 各变量间的相关矩阵 $(r)$ 。

\begin{tabular}{lllll}
\hline & $\overline{\mathbf{x}} \pm \mathbf{s}$ & $\mathbf{1}$ & $\mathbf{2}$ & $\mathbf{3}$ \\
\hline 1特质焦虑 & $68.19 \pm 19.25$ & 1 & & \\
2无法忍受不确定性 & $52.78 \pm 24.60$ & $0.288^{* *}$ & 1 & $-0.476^{* *}$ \\
3掌控感 & $59.60 \pm 17.48$ & $-0.270^{* *}$ & $0.357^{* *}$ & 1 \\
4状态焦虑 & $39.81 \pm 27.57$ & $0.617^{* *}$ & $-0.389^{* *}$ & 1 \\
\hline
\end{tabular}

采用Pearson相关检验各变量间的相关关系。如表1所 示, 特质焦虑、无法忍受不确定性与状态焦虑三者两两之 间均呈显著正相关，而特质焦虑、无法忍受不确定性、状 态焦虑均与掌控感呈显著负相关。

\section{2. 独立中介效应分析}

\subsection{1. 特质焦虑-无法忍受不确定性-状态焦虑路径分析}

参照Hayes提出的Bootstrap方法进行无法忍受不确定 性在特质焦虑与状态焦虑关系中中介效应检验[27]。选择 模型 4 , 样本量 5000 , 在 $95 \%$ 置信区间下, 以特质焦虑为 自变量 $X$, 状态焦虑为因变量 $Y$, 无法忍受不确定性为中 介变量M。结果发现, 无法忍受不确定性的中介检验的结 果没有包含 $0(B=0.0804, S E=0.0508,95 \%$ 置信区间 $=[0.0091,0.2184])$, 表明无法忍受不确定性的中介效应显 著。此外, 控制了中介变量无法忍受不确定性之后, 自变 量特质焦虑对因变量状态焦虑的影响显著 $(B=0.8032$, $S E=0.1226,95 \%$ 置信区间 $=[0.5595,1.0468])$ 。因此无法忍 受不确定性在特质焦虑对状态焦虑的影响中发挥了部分 中介作用, 相对中介效应, 即中介效应占总效应的比例为 $9.10 \%$ 。

\subsection{2. 特质焦虑-掌控感-状态焦虑路径分析}

以掌控感为中介变量 $\mathrm{M}$, 其他步骤与 3.2 .1 过程相同。 结果发现, 掌控感的中介检验的结果没有包含 $0(B=0.0930$, $S E=0.0463,95 \%$ 置信区间 $=[0.0251,0.2140])$, 表明掌控感
的中介效应显著。此外, 控制了中介变量掌控感之后, 自 变量特质焦虑对因变量状态焦虑的影响显著 $(B=0.7906$, $S E=0.1200,95 \%$ 置信区间 $=[0.5521,1.0291])$ 。因此掌控感 在特质焦虑对状态焦虑的影响中发挥了部分中介作用, 相 对中介效应为 $10.53 \%$ 。

\section{3. 链式中介效应分析}

\subsection{1. 特质焦虑-无法忍受不确定性-掌控感-状态焦虑路径 分析}

参照Hayes提出的Bootstrap方法进行无法忍受不确定 性、掌控感在特质焦虑与状态焦虑关系中链式中介效应检 验[27]。选择模型6, 样本量 5000, 在95\%置信区间下, 以 特质焦虑为自变量 $X$, 状态焦虑为因变量 $Y$, 无法忍受不 确定性、掌控感分别为中介变量M1、M2。由表2、3可知, 无法忍受不确定性、掌控感的中介作用显著, 且中介效应 通过唯一中介链产生: 特质焦虑 $\rightarrow$ 无法忍受不确定性 $\rightarrow$ 掌 控感 $\rightarrow$ 状态焦虑，其相对中介效应为 $3.89 \%$ 。具体路径如 图1所示。

表2 间接效应路径。

\begin{tabular}{ll}
\hline 间接效应 & 路径 \\
\hline 1 & 特质焦虑 $\rightarrow$ 无法忍受不确定性 $\rightarrow$ 状态焦虑 \\
2 & 特质焦虑 $\rightarrow$ 无法忍受不确定性 $\rightarrow$ 掌控感 $\rightarrow$ 状态焦虑 \\
3 & 特质焦虑 $\rightarrow$ 掌控感 $\rightarrow$ 状态焦虑 \\
\hline
\end{tabular}

表3 间接效应值与效果量。

\begin{tabular}{|c|c|c|c|c|c|}
\hline & 效应值 & Boot标准误 & $95 \%$ LLCI & $95 \%$ ULCI & 相对中介效应 \\
\hline 总间接效应 & 0.1205 & 0.0562 & 0.0328 & 0.2575 & $13.64 \%$ \\
\hline 间接效应1 & 0.0461 & 0.0512 & -0.0245 & 0.1820 & $5.22 \%$ \\
\hline 间接效应2 & 0.0344 & 0.0243 & 0.0039 & 0.1161 & $3.89 \%$ \\
\hline 间接效应3 & 0.0401 & 0.0349 & -0.0041 & 0.1476 & $4.54 \%$ \\
\hline
\end{tabular}




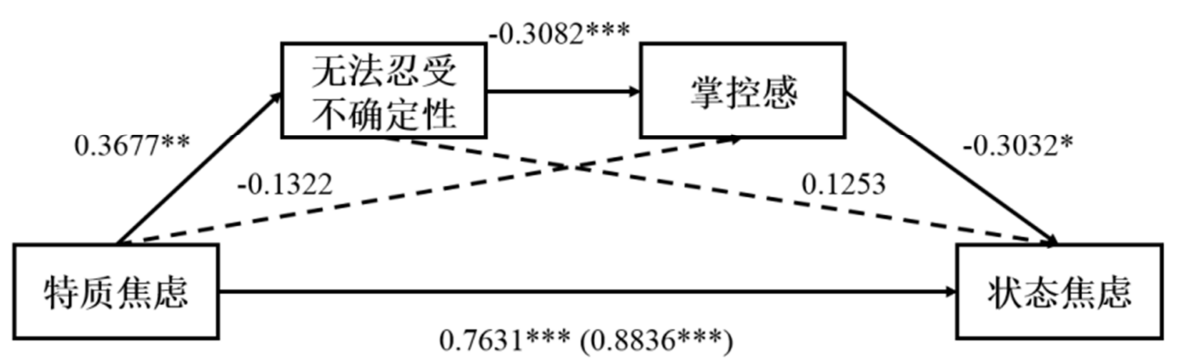

图1 链式中介作用模型。

\subsection{2. 特质焦虑-掌控感-无法忍受不确定性-状态焦虑路径 分析}

以掌控感、无法忍受不确定性分别为中介变量M1、 $\mathrm{M} 2$, 其他步骤与2.3.1过程相同。结果发现, 特质焦虑 $\rightarrow$ 掌控感 $\rightarrow$ 无法忍受不确定性 $\rightarrow$ 状态焦虑路径的间接效应 的95\%置信区间包括0 $(B=0.0186, S E=0.0207,95 \%$ 置信区 间 $=[-0.0079,0.0787])$, 说明特质焦虑不通过路径掌控感 $\rightarrow$ 无法忍受不确定性影响状态焦虑。

\section{4. 讨论}

本研究的主要目的是探究特质焦虑、无法忍受不确定 性以及掌控感如何共同影响状态焦虑的关系。

第一, 本研究发现特质焦虑对状态焦虑具有直接影响, 即高特质焦虑者也会表现出了较高的状态焦虑, 这与以往 的理论与研究结果一致 $[6,28]$, 反映了焦虑的稳定人格特 质倾向与暂时性状态反应之间必然的内在联系。

第二, 无法忍受不确定性和掌控感的单独中介作用表 明, 无法忍受不确定性、掌控感是特质焦虑导致状态焦虑 的中介因素, 即特质焦虑对状态焦虑的影响主要是通过增 加个体的无法忍受不确定性以及降低个体的掌控感实现 的。个体的特质焦虑水平越高, 其无法忍受不确定性越强 或者掌控感越弱, 状态焦虑水平就会越高。

第三, 本研究发现无法忍受不确定性、掌控感在特质 焦虑与状态焦虑间具有链式中介作用。具体表现为: 特质 焦虑通过无法忍受不确定性 $\rightarrow$ 掌控感的链式中介作用这 一间接路径影响个体的状态焦虑。特质焦虑的个体倾向于 把外界广泛的刺激环境知觉为危险的或威胁的 [6]。这是因 为高特质焦虑个体具有高无法忍受不确定性, 而正是这种 高无法忍受不确定性使得个体通常倾向于将不确定性情 境或事件进行预期性的灾难化解释, 并夸大预期性灾难发 生的可能性与严重性[11,29]。个体认为在灾难还没来临之 前需要事先想办法应对 [30]。然而高无法忍受不确定性的 个体对问题解决存在消极的自我效能及结果期待 [31]。因 此, 他们会体验到对即将发生的灾难无法应对[32], 即表 现出低掌控感。此时, 个体便会产生较高的焦虑水平。这 一发现揭示了特质焦虑先后通过无法忍受不确定性及掌 控感影响状态焦虑的内部过程。

本研究的理论贡献是, 将特质焦虑与状态焦虑之间的 关系放在一个链式框架中进行解释, 进而发现无法忍受不 确定性和掌控感的中介作用。这个发现的意义在于为通过
提高不确定性的忍受程度以及掌控感来预防与缓解焦虑 提供了直接的实验证据。

\section{5. 结论}

特质焦虑对状态焦虑具有直接影响; 无法忍受不确定 性和掌控感分别在特质焦虑影响状态焦虑的过程中具有 部分中介作用; 同时特质焦虑通过路径无法忍受不确定性 $\rightarrow$ 掌控感、而非掌控感 $\rightarrow$ 无法忍受不确定性的链式中介作 用影响状态焦虑。

\section{致谢}

本文为国家自然科学基金项目（71871124）及国家社 科重点基金项目（20AZD085）的阶段性成果之一。

\section{参考文献}

[1] Bekker HL, Legare F, Stacey D, et al. Is anxiety a suitable measure of decision aid effectiveness: a systematic review? Patient Education \& Counseling, 2003, 50(3): 255-262.

[2] 张瑜, 郑希付, 黄珊珊, 等. 不同线索下特质焦虑个体的返 回抑制. 心理学报, 2013, 45(4): 446-452.

[3] 徐贵雄. 焦虑程度的客观判别——基于fNIRS技术的应用. 华南师范大学, 2015 .

[4] 唐海波, 广春霞, 姚树桥. 焦虑障碍的易感性-应激发展观 综述. 中国临床心理学杂志, 2008, 16(2): 222-224.

[5] Barlow DH. Anxiety and its disorders: The nature and treatment of anxiety panic. New York: Guiford Press, 1988.

[6] Spielberger CD. Notes and comments trait-state anxiety and motor behavior. Journal of Motor Behavior, 1971, 3(3): 265-279.

[7] 李志勇, 吴明证, 陶伶, 等. 大学生自尊、无法忍受不确定 性、职业决策困难与就业焦虑的关系. 中国临床心理学杂 志, 2012, 20(4): 564-566.

[8] 黄仁辉, 李洁, 李文虎. 不确定性容忍度对风险偏好的影 响及其情景依赖性. 心理科学, 2014(6): 1302-1307.

[9] Boelen PA, Reijntjes A. Intolerance of uncertainty and social anxiety. Journal of Anxiety Disorders, 2009, 23(1): 130-135. 
[10] Yao N, Qian M, Jiang Y, Elhai J. The Influence of Intolerance of Uncertainty on Anxiety and Depression Symptoms in Chinese-speaking Samples: Structure and Validity of The Chinese Translation of The Intolerance of Uncertainty Scale. Journal of Personality Assessment, 2021, 103(3): 406-415.

[11] 李志勇, 吴明证, 欧阳儒阳, 等. 大学生无法忍受不确定性 与社交焦虑的关系:序列中介效应分析. 中国临床心理学杂 志, 2014, 22(1): 167-169.

[12] 李洁, 张乐君, 温兆优, 黄仁辉, 董圣鸿. 父母心理控制对 流动儿童社交焦虑的影响: 自尊和无法忍受不确定性的链 式中介作用。中国临床心理学杂志，2021，29(1): 139-143+103.

[13] Koerner N, Dugas MJ. An investigation of appraisals in individuals vulnerable to excessive worry: The role of intolerance of uncertainty. Cognitive Therapy \& Research, 2008, 32(5): 619-638.

[14] 崔慧茹, 李伟, 庞娇艳, 李惠, 李春波. 焦虑障碍患者无法 忍受不确定性水平及相关因素. 上海交通大学学报(医学 版), 2020, 40(3): 346-350.

[15] Folkman S, Lazarus RS, Gruen RJ, et al. Appraisal, coping, health status, and psychological symptoms. Journal of Personality and Social Psychology, 1986, 50(3): 571-579.

[16] Pearlin LI, Schooler C. The structure of coping. Journal of Health \& Social Behavior, 1978, 19(1): 2-21.

[17] Beck AT, Emery G, Greenberg RL. Anxiety disorders and phobias: A cognitive perspective. Psychosomatics, 2008, 27(2): 155 .

[18] 杨萍. 焦虑的发展: 控制在早期环境中的作用. 社会心理 科学, 1999(4): 10-12.

[19] Gallagher S, Trigg D. Agency and anxiety: Delusions of control and loss of control in schizophrenia and agoraphobia. Frontiers in Human Neuroscience, 2016, 10: 459.

[20] Hofmann SG. Perception of control over anxiety mediates the relation between catastrophic thinking and social anxiety in social phobia. Behaviour Research \& Therapy, 2005, 43(7): 885-895.
[21] 黄韵榛，蒋婧琪，姚泥沙，赵楠，钱铭怡. 自我聚焦注意与 社交焦虑: 控制感的中介作用. 中国临床心理学杂志, 2019, 27(6): 1232-1236.

[22] 李文利, 钱铭怡. 状态特质焦虑量表中国大学生常模修订. 北京大学学报:自然科学版, 1995, 31(1): 108-114.

[23] Lozano LM, Garcíacueto E, Muñiz J. Effect of the number of response categories on the reliability and validity of rating scales. Methodology, 2008, 4(2): 73-79.

[24] 陈欣欣, 于洪彦. 等级评定量表的最佳反应项数目:来自眼 动研究的证据. 心理与行为研究, 2014, 12(4): 551-557.

[25] 乔柱，杨国盛，Amol Ghorpade，等. 双极主观等级量表的 级别选取. 机械设计与研究, 2009, 25(4): 70-72.

[26] Mccormack HM, Horne DJ, Sheather S. Clinical applications of visual analogue scales: a critical review. Psychological Medicine, 1988, 18(4): 1007-1019.

[27] Hayes AF. Methodology in the social sciences. Introduction to mediation, moderation, and conditional process analysis: A regression-based approach. New York: Guilford Press, 2013

[28] Otto MW. Anxiety and its disorders: The nature and treatment of anxiety and panic. Clinical Psychology Review, 2002, 26(1): 105-106.

[29] 李志勇, 王大鹏，吴明证，等. 无法忍受不确定性与担忧、 焦虑的关系：问题取向的中介作用. 中国临床心理学杂志, 2015, 23(5): 808-811.

[30] Baron-Cohen S, Wheelwright S. The empathy quotient: an investigation of adults with Asperger syndrome or high functioning autism, and normal sex differences. Journal of Autism and Developmental Disorders, 2004, 34: 163-175.

[31] 张国华, 戴必兵. 无法忍受不确定性研究进展. 首都师范 大学学报(社会科学版), 2012(2): 124-130.

[32] Dugas MJ, Marchand A, Ladouceur R. Further validation of a cognitive-behavioral model of generalized anxiety disorder: diagnostic and symptom specificity. Journal of Anxiety Disorders, 2005, 19(3): 329-343. 